INSTITUT NATIONAL DE RECHERCHE EN INFORMATIQUE ET EN AUTOMATIQUE

\title{
The Central Limit Theorem for a non linear algorithm based on quantization
}

Vlad Bally

$\mathbf{N}^{\circ} 4629$

Octobre 2002

THÈME 4 



\title{
The Central Limit Theorem for a non linear algorithm based on quantization
}

\author{
Vlad Bally \\ Thème 4 - Simulation et optimisation \\ de systèmes complexes \\ Projet Mathfi \\ Rapport de recherche $\mathrm{n}^{\circ} 4629$ - Octobre 2002 - 23 pages
}

\begin{abstract}
In a serry of papers we have presented an algorithm based on quantization for pricing American options. More generally this amounts to solve numerically an obstacle problem for some semi linear $P D E^{\prime} s$. Our algorithm is based on a Monte Carlo method and so a statistical error comes on. In the present paper we study this error: we prove the Central Limit Theorem for the algorithm and we give evaluations of the variance.. The difficulty comes from the fact that the algorithm is not linear. On the other hand an interesting problem is to control the behaviour of the variance of the algorithm as the complexity increases.. It turns out that the variance does not blow up if the time discretization step and the space discretization step tend to zero
\end{abstract}

Key-words: Numerical Probability, Quantization of random variables, Optimal stopping, Semi linear $P D E^{\prime} s$, Central Limit Theorem, American options 


\section{Le théorème de limite centrale pour un algorithme non linéaire basé sur la quantification}

Résumé : Nous avons déjà présenté dans une série d'articles un algorithme basé sur la quantification pour le calcul des prix d'options Américaines. D'une manière plus générale ceci revient à la résolution numérique d'un problème d'obstacles pour une EDP semi-linéaire. Comme notre algorithme est basé sur une méthode de Monte-Carlo, une erreur statistique apparaît. Dans ce papier nous évaluons cette erreur, plus précisement on démontre un théorème de limite centrale pour l'algorithme et on donne une évaluation de la variante. La difficulté vient du fait que notre algorithme est non linéaire. D'autre part il est intéressant de contrôler l'assymptotique de la variante quand la complexité de l'algorithme croit. On démontre que la variante reste bornée quand les pas de discrétisation en temps et en espace tends vers zéro (et donc la complexité de l'algorithme tend vers l'infini.

Mots-clés : Probabilités numériques, quantification des variables aléatoires, arrêt optimal, EDP semi-linéaires, théorème de limite centrale, options américaines 


\section{Introduction}

It is well known that the Monte Carlo method is an alternative to the deterministic algorithms in order to compute the solution of linear $P D E^{\prime} s$. This is especially interesting in dimension larger then three or four when the deterministic methods become difficult to implement. The foundation of the Monte Carlo method is to represent the solution of the $P D E$ as the expectation (by means of the Fayneman Kac formula) and then to replace the mathematical expectation by an empirical expectation. Unfortunately this approach fails if we deal with non - linear $P D E^{\prime} s$ because in this case the solution can not be represented as an expectation. But recently Pardoux and Peng (see $[P . P],[E l-K . P . Q] \ldots$ ) have given a generalization of the Feyneman Kac formula by means of Backward Stochastic Differential Equations and moreover, in $[E l-K$ and all] (see also [B.C.F.El $-K]$ ) one obtatins such a probabilistic representation for $P D E^{\prime} s$ with obstacle. Then one may employ a Dynamical Programing Principle in order to write down an approximation scheme for the solution of a semi linear $P D E$ respectively a semi linear $P D E$ with free boundary.. The delicate point in implementing such an approximation scheme is that one has to compute a large number of conditional expectations which is far from being trivial. Several methods for solving such a problem has been proposed in the last years, mainly motivated by mathematical finance problems such as pricing American options based on a large number of assets (see $[B . G],[L . S],[L . R], \ldots)$.

In $[B . P .1],[B . P .2]$ and $[B . P . P]$ we have presented a new method for solving this problem, based on quantization. This amounts to replace the diffusion process underling the $P D E$ by the Euler scheme first and then to project the Eule scheme on a given grid (see Section 3 here for a detailed description). Then we have to compute quantities of the type $E\left(\phi\left(X_{k+1}\right) \mid X_{k}\right)$ where $X_{k}$ are now discrete random variables (the projection of the Euler scheme at time $t_{k}$ ). Here comes on the Monte Carlo method because we replace the exact transition probabilities by some empirical frequencies obtained by the simulation of the underlying diffusion process (in fact of the Euler Scheme corresponding to the diffusion). As in the case of the standard Monte Carlo method, the Low of Large Numbers guaranties the convergence when the size of the sample goes to infinity. In this paper our problem is to evaluate the speed of convergence, so to obtain a Central Limit Theorem for our algorithm. (Such a program has already been achieved for the Langstaff Schwarz algorithm in [Lamberton...])

It turns out that the problem at hand appears as a particular case of the following more general problem. On a probability space $(\Omega, F, P)$ we consider a sequence of random variables $X_{k}, k=0, \ldots n$, which take a finite number $N_{k}$ of values, i.e $X_{k} \in \Gamma_{k}=\left\{x_{k}^{1}, \ldots, x_{k}^{N_{k}}\right\} \subseteq E$, where $E$ is some abstract space. We assume that $X_{0}=x_{0}$ is deterministic. We consider the conditional expectations

$$
\begin{aligned}
\pi_{i j}^{k} & =\frac{P\left(X_{k+1}=x_{k+1}^{j}, X_{k}=x_{k}^{i}\right)}{P\left(X_{k}=x_{k}^{i}\right)}, \quad i=1, \ldots, N_{k}, j=1, \ldots, N_{k+1} \\
p_{k}^{i} & =P\left(X_{k}=x_{k}^{i}\right), \quad i=1, \ldots, N_{k} .
\end{aligned}
$$

$\mathrm{RR} \mathrm{n}^{\circ} 4629$ 
Moreover, we consider a sample $\left(X_{k}^{l}\right)_{k \geq 0}, l=1, \ldots, M$ of $\left(X_{k}\right)_{k \geq 0}$ and we define the empirical conditional expectations

$$
\bar{\pi}_{i j}^{k}=\frac{\sum_{l=1}^{M} 1_{\left\{X_{k+1}^{l}=x_{k+1}^{j}\right\}} 1_{\left\{X_{k}^{l}=x_{k}^{i}\right\}}}{\sum_{l=1}^{M} 1_{\left\{X_{k}^{l}=x_{k}^{i}\right\}}}, \quad i=1, \ldots, N_{k}, j=1, \ldots, N_{k+1}
$$

with the convention that $\bar{\pi}_{i j}^{k}=0$ if

$$
s_{k}^{i}=: \sum_{l=1}^{M} 1_{\left\{X_{k}^{l}=x_{k}^{i}\right\}}=0 .
$$

Given a final condition $g: R^{d} \rightarrow R$ and some coefficients $F_{k}: E \times R \rightarrow R$ we consider the dynamical programing algorithm

$$
\begin{aligned}
& \widehat{u}_{n}\left(x_{n}^{i}\right)=g\left(x_{n}^{i}\right) \quad i=1, \ldots, N_{n} \\
& \widehat{u}_{k}\left(x_{k}^{i}\right)=\sum_{j=1}^{N_{k+1}} F_{k+1}\left(x_{k+1}^{j}, \widehat{u}_{k+1}\left(x_{k+1}^{j}\right)\right) \pi_{i j}^{k} \quad i=1, \ldots, N_{k} .
\end{aligned}
$$

This is our algorithm. The corresponding algorithm using the empirical conditional probabilities is

$$
\begin{aligned}
& \bar{u}_{n}\left(x_{n}^{i}\right)=g\left(x_{n}^{i}\right) \\
& \bar{u}_{k}\left(x_{k}^{i}\right)=\sum_{j=1}^{N_{k+1}} F_{k}\left(x_{k+1}^{j}, \bar{u}_{k+1}\left(x_{k+1}^{j}\right)\right) \bar{\pi}_{i j}^{k} .
\end{aligned}
$$

The law of large numbers gives $\bar{\pi}_{i j}^{k} \rightarrow \pi_{i j}^{k}$ as $M \rightarrow \infty$ and consequently, $\bar{u}_{k}\left(x_{k}^{i}\right) \rightarrow \widehat{u}_{k}\left(x_{k}^{i}\right)$. Our aim is to establish a Central Limit Theorem corresponding to this convergence.

Over all the paper we assume that

$$
\left|F_{k}(x, y)-F_{k}\left(x, y^{\prime}\right)\right| \leq\left[F_{k}\right]_{1}\left|y-y^{\prime}\right| .
$$

We also denote $[F]_{1}=\max \left[F_{k}\right]_{1}$. Note that there is no blondeness assumption in our hypothesis. This is because we are interested in the values of the functions at hand in a finite number of points, namely in $x_{k}^{i}, k=1, n, i=1, N_{k}$. So we denote

$$
\|\phi\|_{\Gamma, \infty}=\max _{k=1, n} \max _{i=1, N_{k}}\left|\phi\left(x_{k}^{i}\right)\right| \quad \text { and } \quad\|\phi\|_{\infty}=\sup _{x \in R^{d}}|\phi(x)| .
$$

In Section 2 we prove that under the above hypothesis the $C L T$ holds, that is

$$
\sqrt{M}\left(\bar{u}_{0}\left(x_{0}\right)-\widehat{u}_{0}\left(x_{0}\right)\right) \rightarrow N\left(0, \sigma_{n, N}\right)
$$


where $\sigma_{n, N}$ is a constant which depends on the number $n$ of steps in our algorithm and on the maximal number of points on each layer, that is $N=\max _{k=0, n} N_{k}$.

The interesting problem now concerns the behavior of the variance $\sigma_{n, N}$ as $n, N \rightarrow \infty$. In the general frame described before we can say nothing more than $\sigma_{n, N} \leq C n$, so the variance may blow up as $n \rightarrow \infty$. In order to obtain more precise results we have to restrict ourself to a Markovian frame: we assume that the random variables $\mathrm{XX}_{k}, k=1, \ldots n$ are obtained from a Markov chain $\bar{X}_{k}, k=1, \ldots, n$ (for example the Euler Scheme of a diffusion process) by projection on the grid $\Gamma_{k}$ (see Section 3 ; this is the quantization procedure employed in [B.P.1], [B.P.2], [B.P.P]). Then we obtain quite precise evaluations of $\sigma_{n, N}$ (see Proposition 4 ) which essentially guarantees that under reasonable hypothesis the variance does not blow up.

In Section 3 we consider some specific functions $F_{k}$ which correspond to the following two concrete examples. First of all we set up a dynamical programing algorithm for solving numerically the semi - linear $P D E$

$$
(\partial+L) u+f\left(t, x, u_{t}\right)=0, \quad u_{T}=g .
$$

In order to construct such an algorithm one takes (see [B.P.1]) $F_{k}(x, y)=y+\frac{T}{n} f\left(\frac{k T}{n}, x, y\right)$. If $f$ is twice differentiable with respect to $y$ we are able to give very precise evaluations of $\sigma_{n, N}$. We prove that $\underline{\sigma}-C / n \leq \sigma_{n, N} \leq \bar{\sigma}+C / n$ where $\underline{\sigma}$ and $\bar{\sigma}$ have the following explicit expression (see Section 3.1):

$$
\bar{\sigma}=e^{T[f]_{1}} P_{T} g^{2}\left(x_{0}\right)-u_{0}\left(x_{0}\right)+[f]_{1} \int_{0}^{T} e^{t[f]_{1}} P_{t}\left(u_{t}^{2}+u_{t} f\left(t, ., u_{t}\right)\right)\left(x_{0}\right) d t
$$

where $P_{t}$ is the semigroup of infinitesimal operator $L$ and $u$ is the solution of the PDE. $\underline{\sigma}$ has the same expression with $e^{-t[f]_{1}}$ instead of $e^{t[f]_{1}}$.

The second problem - which is the really interesting one - concerns the semi - linear $P D E$ with reflection

$$
\max \left\{h_{t},(\partial+L) u+f\left(t, x, u_{t}\right)\right\}=0, \quad u_{T}=h_{T} .
$$

Now $F_{k}(x, y)=\max \left\{h\left(t_{k}, x\right), y+\frac{T}{n} f\left(\frac{k T}{n}, x, y\right)\right\}$. The problem is much more "irregular" because $y \rightarrow F_{k}(x, y)$ is no more continuously differentiable. In order to handle this irregularity we have to split the error $\bar{u}_{0}\left(x_{0}\right)-\widehat{u}_{0}\left(x_{0}\right)=\varepsilon_{n, N}^{M}+m_{n, N}^{M}$ where $m_{n, N}^{M}(\omega)$ is a random variable which is dominated by $\frac{C}{n}$ for every $\omega$ and every $M$. Since this correction does not depend on $\omega$ and $M$ it does not significantly contribute to the statistical error (we may consider it as a part of the analytical error $u-\widehat{u}$ which is of order $\frac{1}{n}$ as well). So the statistical error is described by $\varepsilon_{n, N}^{M}$ and we prove that $\sqrt{M} \varepsilon_{n, N}^{M} \rightarrow N\left(0, \sigma_{n, N}\right)$ and $\sigma_{n, N} \leq \frac{C}{\sqrt{n}}+C \bar{\sigma}$. We are not able to minorate the variance $\sigma_{n, N}$ in this case. 


\section{The CLT in the abstract frame}

\section{$2.1 \quad$ Notation}

We denote by $\bar{E}_{i}^{k}$ the expectation with respect to the empirical probability measure $\bar{\pi}_{i j}^{k}, j=$ $1, \ldots, N_{k+1}$ that is

$$
\bar{E}_{i}^{k} \phi=\sum_{j=1}^{N_{k+1}} \phi\left(x_{k+1}^{j}\right) \bar{\pi}_{i j}^{k}=\frac{1}{s_{k}^{i}} \sum_{l=1}^{M} \phi\left(X_{k+1}^{l}\right) 1_{\left\{X_{k}^{l}=x_{k}^{i}\right\}}
$$

and we also define

$$
E_{i}^{k} \phi=\sum_{j=1}^{N_{k+1}} \phi\left(x_{k+1}^{j}\right) \pi_{i j}^{k}=\frac{1}{p_{k}^{i}} E\left(\phi\left(X_{k+1}\right) 1_{\left\{X_{k}=x_{k}^{i}\right\}}\right)
$$

Moreover we denote

$$
G_{k}=\sigma\left(X_{k}^{1}, \ldots, X_{k}^{M}, X_{k}\right) \quad \text { and } \quad E_{G_{k}}(\Phi)=: E\left(\Phi \mid G_{k}\right)
$$

We have the following key identity:

$$
1_{\left\{X_{k}^{l}=x_{k}^{i}\right\}} E_{G_{k}}\left(\phi\left(X_{k+1}^{l}\right)\right)=1_{\left\{X_{k}^{l}=x_{k}^{i}\right\}} E\left(\phi\left(X_{k+1}^{l}\right) \mid X_{k}^{l}\right)=1_{\left\{X_{k}^{l}=x_{k}^{i}\right\}} E_{i}^{k} \phi .
$$

Moreover, for $p>k$ we define by recurrence the transition probability densities from $\Gamma_{k}$ to $\Gamma_{p+1}$

$$
\begin{aligned}
& \pi_{i j}^{k, p}= \sum_{r=1}^{N_{p-1}} \pi_{i r}^{k, p-1} \pi_{r j}^{p}, \quad \text { with } \quad \pi_{i j}^{k, k}=\pi_{i j}^{k} \\
& \text { and } \\
& \bar{\pi}_{i j}^{k, p}=\sum_{r=1}^{N_{p-1}} \bar{\pi}_{i r}^{k, p-1} \bar{\pi}_{r j}^{p}, \quad \text { with } \quad \bar{\pi}_{i j}^{k, k}=\bar{\pi}_{i j}^{k} .
\end{aligned}
$$

Then we denote

$$
E_{i}^{k, p} \phi=\sum_{j=1}^{N_{p+1}} \phi\left(x_{p+1}^{j}\right) \pi_{i j}^{k, p}, \quad \bar{E}_{i}^{k, p} \phi=\sum_{j=1}^{N_{p+1}} \phi\left(x_{p+1}^{j}\right) \bar{\pi}_{i j}^{k, p} .
$$

It is easy to check that we have the following recurrence formulas

$$
E_{i}^{k, p} \phi=E_{i}^{k, p-1} E_{.}^{p} \phi, \quad \bar{E}_{i}^{k, p} \phi=\bar{E}_{i}^{k, p-1} \bar{E}_{.}^{p} \phi
$$

where we make the slight abuse of notation $\left(E^{p} \phi\right)\left(x_{p}^{i}\right)=: E_{i}^{p} \phi$. 
The starting time $k=0$ plays a particular roll because $X_{0}=x_{0}$ is deterministic. In order to employ unitary notation we assume that a grid $\Gamma_{0}=\left\{x_{0}^{1}, \ldots, x_{0}^{N_{0}}\right\}$ is given and we denote by $i_{0}$ the index such that $x_{0} \in x_{0}^{i_{0}}$ (in fact one may take $N_{0}=1$ and $i_{0}=1$ ) Then it is easy to check by induction that

$$
\pi_{i_{0}, j}^{0, p}=P\left(X_{p+1}=x_{p+1}^{j}\right)=p_{p+1}^{j} \quad \text { and } \quad \bar{\pi}_{i_{0}, j}^{0, p}=\frac{s_{p+1}^{j}}{M}=\frac{1}{M} \sum_{l=1}^{M} 1_{\left\{X_{p+1}^{l}=x_{p+1}^{j}\right\}} .
$$

This is the effect of the composition of the transition probabilities $\pi_{i j}^{k, k+1}, k=0, \ldots, p-1$ (respectively $\bar{\pi}_{i j}^{k, k+1}, k=0, \ldots, p-1$ ). Note that the composition of the transition probabilities $\pi_{i j}^{k, k+1}, k=q, \ldots, p$ does not give such an explicit formula because, for $q>0, X_{q}$ may take several different values.

Finally, given a sequence of functions $H_{k}: E \rightarrow R, k=0, \ldots, n$, we define

$$
\begin{aligned}
\pi_{H, i j}^{k}= & H_{k+1}\left(x_{k+1}^{j}\right) \pi_{i j}^{k}, \quad E_{H, i}^{k} \phi=: \sum_{j=1}^{N_{k+1}} \phi\left(x_{k+1}^{j}\right) \pi_{H, i j}^{k}=E_{i}^{k}\left(H_{k+1} \phi\right) \\
& \text { and } \\
\bar{\pi}_{H, i j}^{k}= & H_{k+1}\left(x_{k+1}^{j}\right) \bar{\pi}_{i j}^{k}, \quad \bar{E}_{H, i}^{k} \phi=: \sum_{j=1}^{N_{k+1}} \phi\left(x_{k+1}^{j}\right) \bar{\pi}_{H, i j}^{k}=\bar{E}_{i}^{k}\left(H_{k+1} \phi\right) .
\end{aligned}
$$

Using the same recurrence procedure as above we define

$$
\begin{aligned}
& \pi_{H, i j}^{k, p}=\sum_{r=1}^{N_{p-1}} \pi_{H, i r}^{k, p-1} \pi_{H, r j}^{p}, \quad \text { with } \quad \pi_{H, i j}^{k, k}=\pi_{H, i j}^{k} \\
& \text { and } \\
& \bar{\pi}_{H, i j}^{k, p}=\sum_{r=1}^{N_{p-1}} \bar{\pi}_{H, i r}^{k, p-1} \bar{\pi}_{H, r j}^{p}, \quad \text { with } \quad \bar{\pi}_{H, i j}^{k, k}=\bar{\pi}_{H, i j}^{k} .
\end{aligned}
$$

Moreover, $E_{H, i}^{k, p} \phi=: \sum_{j=1}^{N_{p+1}} \phi\left(x_{p+1}^{j}\right) \pi_{H, i j}^{k, p}$ and $\bar{E}_{H, i}^{k, p} \phi=: \sum_{j=1}^{N_{p+1}} \phi\left(x_{p+1}^{j}\right) \bar{\pi}_{H, i j}^{k, p}$. Clearly $E_{H, i}^{k, p}$ (respectively $\bar{E}_{H, i}^{k, p}$ ) verifies the same recurrence relations as $E_{i}^{k, p}$ (respectively $\bar{E}_{i}^{k, p}$ ). One also has

$$
\begin{aligned}
\pi_{H, i j}^{k, p} \leq & c_{k, p}(H) \pi_{i j}^{k, p} \text { and } \bar{\pi}_{H, i j}^{k, p} \leq c_{k, p}(H) \bar{\pi}_{i j}^{k, p} \\
& w i t h \\
c_{k, p}(H)= & \left\|H_{k}\right\|_{\Gamma, \infty} \times \ldots \times\left\|H_{p}\right\|_{\Gamma, \infty} .
\end{aligned}
$$




\subsection{The Central Limit Theorem}

In this section we prove the Central Limit Theorem in the abstract frame. We assume first (we remove this restriction at the end of this section) that the coefficients $F_{k}$ are differentiable with respect to $y$ and $\frac{\partial F_{k}}{\partial y}$ is Holder continuous of index $\alpha>0$, i.e

$$
\left|\frac{\partial F_{k}}{\partial y}(x, y)-\frac{\partial F_{k}}{\partial y}\left(x, y^{\prime}\right)\right| \leq C\left|y-y^{\prime}\right|^{\alpha} .
$$

Under this hypothesis we will prove that the statistical error of our algorithm

$$
\Delta_{0}^{i_{0}}=: \bar{u}_{0}\left(x_{0}\right)-\widehat{u}_{0}\left(x_{0}\right)
$$

verifies the $C L T$. The key step is to prove that

$$
\sqrt{M}\left(\Delta_{0}^{i_{0}}-\widehat{\Delta}\right) \rightarrow 0 \text { in probability }
$$

where

$$
\begin{aligned}
\widehat{\Delta} & =\frac{1}{M} \sum_{l=1}^{M} Z_{l} \text { with } \\
Z_{l} & =\sum_{k=1}^{n-1} \sum_{i=1}^{N_{k}} \pi_{F^{1}, i_{0}, i}^{0, k-1} \frac{1}{p_{k}^{i}}\left(\widehat{F}_{k+1}\left(X_{k+1}^{l}\right)-E_{i}^{k} \widehat{F}_{k+1}\right) 1_{\left\{X_{k}^{l}=x_{k}^{i}\right\}} \\
\widehat{F}_{k}(x) & =F_{k}\left(x, \widehat{u}_{k}(x)\right) \quad \text { and } \quad F_{k}^{1}(x)=\frac{\partial F_{k}}{\partial y}\left(x, \widehat{u}_{k}(x)\right)
\end{aligned}
$$

Clearly $Z_{1}, \ldots, Z_{M}$ are independent random variables which are identically distributed and have zero mean (see (6)) so the standard $C L T$ gives

$$
\sqrt{M} \times \widehat{\Delta}=\frac{1}{\sqrt{M}} \sum_{l=1}^{M} Z_{l} \rightarrow \Theta \sim N\left(0, \sigma_{n, N}\right)
$$

with

$$
\sigma_{n, N}^{2}=E|Z|^{2}
$$

where $Z$ is defined in the same way as $Z_{l}$ with $X$ instead of $X^{l}$.

Proposition 1 Assume that (9) hold true. Then

$$
\sqrt{M} \times\left(\bar{u}_{0}\left(x_{0}\right)-\widehat{u}_{0}\left(x_{0}\right)\right) \rightarrow \Theta \sim N\left(0, \sigma_{n, N}\right) \text { in low. }
$$

Proof. As a consequence of (12) it will suffice to prove (10). The proof goes through several steps. 
Step 1. We denote $\Delta_{k}^{i}=: \bar{u}_{k}\left(x_{k}^{i}\right)-\widehat{u}_{k}\left(x_{k}^{i}\right)$. The purpose of this step is to prove that, for every $\varepsilon>0$

$$
M^{\frac{1}{2}-\varepsilon}\left|\Delta_{k}^{i}\right| \rightarrow 0 \quad \text { in probability. }
$$

We write

$$
\begin{aligned}
\Delta_{k}^{i} & =\bar{E}_{i}^{k}\left(F_{k+1}\left(., \bar{u}_{k+1}(.)\right)\right)-E_{i}^{k}\left(F_{k+1}\left(., \widehat{u}_{k+1}(.)\right)\right) \\
& =\bar{E}_{i}^{k}\left(F_{k+1}\left(., \bar{u}_{k+1}(.)\right)-F_{k+1}\left(., \widehat{u}_{k+1}(.)\right)\right)+\rho_{k}^{i} \text { with } \\
\rho_{k}^{i} & =\bar{E}_{i}^{k} \widehat{F}_{k+1}-E_{i}^{k} \widehat{F}_{k+1} .
\end{aligned}
$$

Since $\Delta_{n}=0$,

$$
\begin{aligned}
\left|\Delta_{k}\right| & \leq\left[F_{k+1}\right]_{1} \bar{E}^{k}\left|\Delta_{k+1}\right|+\left|\rho_{k}\right| \leq \ldots \leq \sum_{p=k+1}^{n-1} \Pi_{j=k+1}^{p}\left[F_{j}\right]_{1} \bar{E}^{k, p-1}\left|\rho_{p}\right| \\
& \leq C \max _{p=k+1, n-1} \max _{i=1, N_{p}}\left|\rho_{p}^{i}\right|
\end{aligned}
$$

so we have to prove that

$$
M^{\frac{1}{2}-\varepsilon}\left|\rho_{k}^{i}\right| \rightarrow 0 \quad \text { in probability. }
$$

In view of (5) and of (6)

$$
\rho_{k}^{i}=\frac{1}{s_{k}^{i}} \sum_{l=1}^{M}\left(\widehat{F}_{k+1}\left(X_{k+1}^{l}\right)-E_{k}^{i} \widehat{F}_{k+1}\right) 1_{\left\{X_{k}^{l}=x_{k}^{i}\right\}}=\frac{1}{s_{k}^{i}} \sum_{l=1}^{M}\left(\widehat{F}_{k+1}\left(X_{k+1}^{l}\right)-E_{G_{k}} \widehat{F}_{k+1}\left(X_{k+1}^{l}\right)\right) 1_{\left\{X_{k}^{l}=x_{k}^{i}\right\}} .
$$

Note that the terms in the above sum are ortogonal under $E_{G_{k}}$. It follows that

$$
\begin{aligned}
E_{G_{k}}\left|\rho_{k}^{i}\right|^{2} & =\frac{1}{\left|s_{k}^{i}\right|^{2}} \sum_{l=1}^{M} E_{G_{k}}\left(\widehat{F}_{k+1}\left(X_{k+1}^{l}\right)-E_{G_{k}} \widehat{F}_{k+1}\left(X_{k+1}^{l}\right)\right)^{2} 1_{\left\{X_{k}^{l}=x_{k}^{i}\right\}} \\
& \leq \frac{1}{s_{k}^{i}}\left\|\widehat{F}_{k+1}\right\|_{\Gamma, \infty}^{2} .
\end{aligned}
$$

We write now

$$
\begin{aligned}
P\left(M^{\frac{1}{2}-\varepsilon}\left|\rho_{k}^{i}\right|\right. & \geq \delta)=P\left(M\left|\rho_{k}^{i}\right|^{2} \geq M^{2 \varepsilon} \delta^{2}\right)=P\left(\frac{M}{s_{k}^{i}} \times s_{k}^{i}\left|\rho_{k}^{i}\right|^{2} \geq M^{2 \varepsilon} \delta^{2}\right) \\
& \leq P\left(\frac{M}{s_{k}^{i}} \geq \frac{2}{p_{k}^{i}}\right)+P\left(\frac{2}{p_{k}^{i}} \times s_{k}^{i}\left|\rho_{k}^{i}\right|^{2} \geq M^{2 \varepsilon} \delta^{2}\right) \\
& =P\left(\frac{s_{k}^{i}}{M} \leq \frac{p_{k}^{i}}{2}\right)+P\left(s_{k}^{i}\left|\rho_{k}^{i}\right|^{2} \geq M^{2 \varepsilon} \delta^{2} p_{k}^{i} / 2\right) .
\end{aligned}
$$


By the law of large numbers $\frac{s_{k}^{i}}{M} \rightarrow p_{k}^{i}$ so the first term converges to zero. We use Chebyshev's inequality and (15) in order to dominate the second term by

$$
\frac{2}{M^{2 \varepsilon} \delta^{2} p_{k}^{i}} E\left(s_{k}^{i} E_{G_{k}}\left|\rho_{k}^{i}\right|^{2}\right) \leq \frac{C}{M^{2 \varepsilon}} \rightarrow 0
$$

and so the proof of (13) is completed.

Step 2. Linearization. The aim of this step is to prove that

$$
\sqrt{M}\left(\Delta_{0}^{i_{0}}-\sum_{k=1}^{n-1} \bar{E}_{F^{1}, i_{0}}^{0, k-1} \rho_{k}\right) \rightarrow 0 \quad \text { in probability. }
$$

We define

$$
\nu_{k}^{i}=F_{k}\left(x_{k}^{i}, \bar{u}_{k}\left(x_{k}^{i}\right)\right)-F_{k}\left(x_{k}^{i}, \widehat{u}_{k}\left(x_{k}^{i}\right)\right)-F_{k}^{1}\left(x_{k}^{i}\right) \Delta_{k}^{i} .
$$

Using the Holder property of $\partial F_{k} / \partial y$ and the definition of $\Delta_{k}^{i}$ we obtain

$$
\left|\nu_{k}^{i}\right| \leq C\left|\Delta_{k}^{i}\right|^{1+\alpha} .
$$

Then, by (14)

$$
\Delta_{k}^{i}=\bar{E}_{i}^{k} F_{k}^{1} \Delta_{k+1}+\bar{E}_{i}^{k} \nu_{k+1}+\rho_{k}^{i}=\bar{E}_{F^{1}, i}^{k} \Delta_{k+1}+\bar{E}_{i}^{k} \nu_{k+1}+\rho_{k}^{i} .
$$

Since $\bar{u}_{k}=g=\widehat{u}_{k}$ one has $\Delta_{n}=0$. So we iterate the above equation and obtain

$$
\Delta_{0}^{i_{0}}=\sum_{k=1}^{n-1} \bar{E}_{F^{1}, i_{0}}^{0, k-1} \rho_{k}+\sum_{k=1}^{n-1} \bar{E}_{F^{1}, i_{0}}^{0, k-1} \bar{E}^{k} \nu_{k+1}
$$

and so (16) reduces to

$$
\sqrt{M} \sum_{k=1}^{n-1} \bar{E}_{F^{1}, i_{0}}^{0, k-1} \bar{E}^{k} \nu_{k+1} \rightarrow 0 \quad \text { in probability. }
$$

Using (17) and (13) with $\varepsilon=\alpha / 2(1+\alpha)$ we obtain

$$
\begin{aligned}
\sqrt{M}\left|\bar{E}_{F^{1}, i_{0}}^{0, k-1} \bar{E}^{k} \nu_{k+1}\right| & \leq C \sqrt{M} \max _{i=1, N_{k+1}}\left|\nu_{k+1}^{i}\right| \leq C \sqrt{M} \max _{i=1, N_{k+1}}\left|\Delta_{k+1}^{i}\right|^{1+\alpha} \\
& =C \max _{i=1, N_{k+1}}\left(M^{\frac{1}{2}-\varepsilon}\left|\Delta_{k+1}^{i}\right|\right)^{1+\alpha} \rightarrow 0 .
\end{aligned}
$$

and (16) is proved.

Step 3. The aim of this step is to prove that

$$
\sqrt{M}\left(\widehat{\Delta}-\sum_{k=1}^{n-1} E_{F^{1}, i_{0}}^{0, k-1} \rho_{k}\right) \rightarrow 0 .
$$

INRIA 
Recall that

$$
\rho_{k}^{i}=\frac{1}{s_{k}^{i}} \sum_{l=1}^{M}\left(\widehat{F}_{k+1}\left(X_{k+1}^{l}\right)-E_{k}^{i} \widehat{F}_{k+1}\right) 1_{\left\{X_{k}^{l}=x_{k}^{i}\right\}}
$$

Then it is easy to check that

$$
\widehat{\Delta}=\sum_{k=1}^{n-1} E_{F^{1}, i_{0}}^{0, k-1}\left(\frac{s_{k} / M}{p_{k}} \rho_{k}\right)
$$

and consequently

$$
\sqrt{M}\left(\widehat{\Delta}-\sum_{k=1}^{n-1} E_{F^{1}, i_{0}}^{0,-1} \rho_{k}\right)=\sum_{k=1}^{n-1} E_{F^{1}, i_{0}}^{0, k-1}\left(\left(p_{k}-\frac{s_{k}}{M}\right) \frac{\rho_{k} \sqrt{M}}{p_{k}}\right) .
$$

Since $E_{G_{k}}\left|\rho_{k}^{i}\right| \leq C / \sqrt{s_{k}^{i}}$ (see (15)) we dominate the above term by

$$
C \sum_{k=1}^{n-1} E_{F^{1}, i_{0}}^{0, k-1}\left(\left|p_{k}-\frac{s_{k}}{M}\right| \frac{1}{p_{k} \sqrt{s_{k} / M}}\right)
$$

which, by the law of large numbers converges to zero as $M \rightarrow \infty$.

\section{Step 4. Change of probability.}

In view of (16) and (18) it remains to prove that

$$
\sqrt{M}\left(\sum_{k=1}^{n-1} \bar{E}_{F^{1}, i_{0}}^{0, k-1} \rho_{k}-\sum_{k=1}^{n-1} E_{F^{1}, i_{0}}^{0, k-1} \rho_{k}\right) \rightarrow 0 \quad \text { in probability. }
$$

We write

$$
\sqrt{M}\left(\bar{E}_{F^{1}, i_{0}}^{0, k-1} \rho_{k}-E_{F^{1}, i_{0}}^{0, k-1} \rho_{k}\right)=\sqrt{M} \sum_{i=1}^{N_{k}}\left(\bar{\pi}_{F^{1}, i_{0}, i}^{0, k-1}-\pi_{F^{1}, i_{0}, i}^{0, k-1}\right) \rho_{k}^{i}=\sum_{i=1}^{N_{k}} \alpha_{k}^{i} \times \beta_{k}^{i}
$$

with

$$
\alpha_{k}^{i}=\frac{1}{\sqrt{s_{k}^{i} / M}}\left(\bar{\pi}_{F^{1}, i_{0}, i}^{0, k-1}-\pi_{F^{1}, i_{0}, i}^{0, k-1}\right) \quad \text { and } \quad \beta_{k}^{i}=\sqrt{s_{k}^{i}} \rho_{k}^{i}
$$

By (15) $E_{G_{k}}\left|\beta_{k}^{i}\right|^{2} \leq\left\|\widehat{F}_{k}\right\|_{\Gamma, \infty}^{2}$. Using the low of large numbers, for every $p, i, j, \bar{\pi}_{i, j}^{p}-$ $\pi_{i, j}^{p} \rightarrow 0, a . s$. and so $\bar{\pi}_{F^{1}, i_{0}, i}^{0, k-1}-\pi_{F^{1}, i_{0}, i}^{0, k-1} \rightarrow 0$,a.s. Moreover $s_{k}^{i} / M \rightarrow p_{k}^{i} \neq 0, a . s$. and so $\alpha_{k}^{i} \times \beta_{k}^{i} \rightarrow 0$ in probability and the proof is completed.

We consider now the case when $F_{k}$ is just Lipschitz continuous as a function of $y$, for example $F_{k}(x, y)=\max \left\{h\left(t_{k}, x\right), y+\frac{T}{n} f\left(t_{k}, x, y\right)\right\}$ which appears in [B.P.1] (see the following 
section also). In order to handle such functions we proceed by regularization by convolution. Given $\varepsilon>0$ we consider some regularization kernel $\phi_{\varepsilon}$ and we define

$$
F_{k}^{\varepsilon}(x, y)=\int F_{k}(x, z) \phi_{\varepsilon}(y+z) d z .
$$

Then $\left[F_{k}^{\varepsilon}\right]_{1} \leq\left[F_{k}\right]_{1}$ and $\left\|F_{k}^{\varepsilon}-F_{k}\right\|_{\infty} \leq\left[F_{k}\right]_{1} \varepsilon$. We define $\widehat{u}_{k}^{\varepsilon}$ and $\bar{u}_{k}^{\varepsilon}$ to be the functions constructed in the algorithms (2) and (3) with $F_{k}^{\varepsilon}$ instead of $F_{k}$, and $\widehat{\Delta}^{\varepsilon}, Z^{\varepsilon}, \widehat{F}_{k}^{\varepsilon}$ and $F_{k}^{\varepsilon, 1}$ to be defined as in (11), with $F_{k}^{\varepsilon}$ instead of $F_{k}$. We also denote

$$
m_{n, N}^{\varepsilon}(\omega)=\Delta_{0}^{i_{0}}-\Delta_{0}^{\varepsilon, i_{0}}=\left(\bar{u}_{0}-\bar{u}_{0}^{\varepsilon}\right)\left(x_{0}\right)-\left(\widehat{u}_{0}-\widehat{u}_{0}^{\varepsilon}\right)\left(x_{0}\right)
$$

Proposition 2 Assume that the hypothesis (4) holds true. Then for every $\varepsilon>0$

$$
\begin{aligned}
&\left|m_{n, N}^{\varepsilon}(\omega)\right| \leq 2 n[F]_{1}^{n} \varepsilon, \quad \forall \omega \in \Omega, \\
& \text { and } \\
& \sqrt{M}\left(\left(\bar{u}_{0}\left(x_{0}\right)-\widehat{u}_{0}\left(x_{0}\right)\right)-m_{n, N}^{\varepsilon}(\omega)\right) \rightarrow \Theta^{\varepsilon} \sim N\left(0, \sigma_{n, N}^{\varepsilon}\right) \quad \text { in law }
\end{aligned}
$$

with $\sigma_{n, N}^{\varepsilon}=\sqrt{E\left|Z^{\varepsilon}\right|^{2}}$.

Proof. We write

$$
\begin{aligned}
\bar{u}_{k}\left(x_{k}^{i}\right)-\bar{u}_{k}^{\varepsilon}\left(x_{k}^{i}\right)= & \bar{E}_{i}^{k}\left(F_{k+1}\left(., \bar{u}_{k+1}(.)\right)-F_{k+1}^{\varepsilon}\left(., \bar{u}_{k+1}(.)\right)\right)+ \\
& \bar{E}_{i}^{k}\left(F_{k+1}^{\varepsilon}\left(., \bar{u}_{k+1}(.)\right)-F_{k+1}^{\varepsilon}\left(., \bar{u}_{k+1}^{\varepsilon}(.)\right)\right) .
\end{aligned}
$$

Then $\left\|\bar{u}_{k}-\bar{u}_{k}^{\varepsilon}\right\|_{\Gamma, \infty} \leq[F]_{1}\left(\varepsilon+\left\|\bar{u}_{k+1}-\bar{u}_{k+1}^{\varepsilon}\right\|_{\Gamma, \infty}\right)$. It follows that $\left|\left(\bar{u}_{0}-\bar{u}_{0}^{\varepsilon}\right)\left(x_{0}\right)\right|=$ $\left\|\bar{u}_{0}-\bar{u}_{0}^{\varepsilon}\right\|_{\Gamma, \infty} \leq \varepsilon \sum_{p=0}^{n}[F]_{1}^{p} \leq \varepsilon n[F]_{1}^{n}$. In the same way one proves that $\left|\left(\widehat{u}_{0}-\widehat{u}_{0}^{\varepsilon}\right)\left(x_{0}\right)\right|=$ $\left\|\widehat{u}_{0}-\widehat{u}_{0}^{\varepsilon}\right\|_{\Gamma, \infty} \leq \varepsilon n[F]_{1}^{n}$ and so $\left|m_{n, N}^{\varepsilon}(\omega)\right| \leq 2 n[F]_{1}^{n} \varepsilon$. Finally, using the previous theorem $\sqrt{M}\left(\left(\bar{u}_{0}\left(x_{0}\right)-\widehat{u}_{0}\left(x_{0}\right)\right)-m_{n, N}^{\varepsilon}(\omega)\right)=\sqrt{M}\left(\bar{u}_{0}^{\varepsilon}\left(x_{0}\right)-\widehat{u}_{0}^{\varepsilon}\left(x_{0}\right)\right) \rightarrow \Theta^{\varepsilon} \sim N\left(0, \sigma_{n, N}^{\varepsilon}\right)$.

\subsection{Evaluations of the variance}

Let us now give some evaluations of the variance.. Recall that under the hypothesis (4) we have $\pi_{F^{1}, i_{0}, i}^{0, k} \leq \Pi_{j=1}^{k+1}\left[F_{j}\right]_{1} p_{k+1}^{i}$. Then an immediate rough evaluation gives

$$
|Z| \leq 2 \sum_{k=1}^{n-1} \Pi_{i=1}^{k}\left[F_{i}\right]_{1}\left\|F_{k+1}\right\|_{\infty}
$$

and consequently

$$
\sigma_{n, N} \leq 2 \sum_{k=1}^{n-1} \Pi_{i=1}^{k}\left[F_{i}\right]_{1}\left\|F_{k+1}\right\|_{\infty} .
$$

INRIA 
This quantity blows up as $n \rightarrow \infty$, as $C n$ (in reasonable algorithms $\left[F_{i}\right]_{1} \leq 1+\frac{c}{n}$ so that $\Pi_{i=1}^{k+1}\left[F_{i}\right]_{1} \leq \exp (c T)$ ). In the examples we have in mind (see the following section) the variance remains bounded independently of $n$, but in order to obtain such results one has to consider a more restrictive frame, namely a Markovian frame. For the moment we define a "Markov error" and we express our results in terms of this error and, at the end of this section, we give an evaluation of this error in some special case. We assume that a filtration $\mathcal{F}_{k}, k=0, \ldots, n$ is given such that $X_{k}$ is $\mathcal{F}_{k}$ measurable and we define the "Markov error"

$$
\mu_{k}=E\left(\widehat{F}_{k+1}\left(X_{k+1}\right) \mid X_{k}\right)-E\left(\widehat{F}_{k+1}\left(X_{k+1}\right) \mid \mathcal{F}_{k}\right) .
$$

Before giving our result we need to emphasis one more hypothesis. In some cases (see the example in the following section) there exists also a constant $\{F\}_{1}>0$ such that

$$
F_{k}^{1}(x) \geq\left\{F_{k}\right\}_{1}, \forall x
$$

and then $\pi_{F^{1}, i_{0}, i}^{0, k} \geq \Pi_{j=1}^{k+1}\left\{F_{j}\right\}_{1} p_{k+1}^{i}$.

Proposition 3 i) Suppose that (4) holds true. Then

$$
\begin{aligned}
\sigma_{n, N} \leq & \left(\sum_{k=0}^{n-1} \Pi_{r=1}^{k+1}\left[F_{r}\right]_{1}^{2} E\left(\widehat{F}_{k+1}^{2}\left(X_{k+1}\right)-\widehat{u}_{k}^{2}\left(X_{k}\right)\right)\right)^{1 / 2} \\
& +2 \sum_{k=0}^{n} \Pi_{i=1}^{k+1}\left[F_{i}\right]_{1}\left\|F_{k+1}\right\|_{\infty}\left\|\mu_{k}\right\|_{2}
\end{aligned}
$$

ii) Suppose that (21) holds also. Then

$$
\begin{aligned}
\sigma_{n, N} \geq & \left(\sum_{k=0}^{n-1} \Pi_{r=1}^{k+1}\left\{F_{r}\right\}_{1}^{2} E\left(\widehat{F}_{k+1}^{2}\left(X_{k+1}\right)-\widehat{u}_{k}^{2}\left(X_{k}\right)\right)\right)^{1 / 2} \\
& -2 \sum_{k=0}^{n} \Pi_{i=1}^{k+1}\left[F_{i}\right]_{1}\left\|F_{k+1}\right\|_{\infty}\left\|\mu_{k}\right\|_{2} .
\end{aligned}
$$

Proof. We denote

$$
\begin{aligned}
Q^{k} & =\sum_{i=1}^{N_{k}} \pi_{F^{1}, i_{0}, i}^{0, k-1} \frac{1}{p_{k}^{i}}\left(\widehat{F}_{k+1}\left(X_{k+1}\right)-E_{i}^{k} \widehat{F}_{k+1}\right) 1_{\left\{X_{k}=x_{k+}^{i}\right\}} \\
& =\sum_{i=1}^{N_{k}} \pi_{F^{1}, i_{0}, i}^{0, k-1} \frac{1}{p_{k}^{i}}\left(\widehat{F}_{k+1}\left(X_{k+1}\right)-E\left(\widehat{F}_{k+1}\left(X_{k+1}\right) \mid X_{k}\right)\right) 1_{\left\{X_{k}=x_{k+}^{i}\right\}}
\end{aligned}
$$


so that $Z=\sum_{k=0}^{n-1} Q^{k}$. Moreover we define

$$
\begin{aligned}
\bar{Q}^{k}= & \sum_{i=1}^{N_{k}} \pi_{F^{1}, i_{0}, i}^{0, k-1} \frac{1}{p_{k}^{i}}\left(\widehat{F}_{k+1}\left(X_{k+1}\right)-E\left(\widehat{F}_{k+1}\left(X_{k+1}\right) \mid \mathcal{F}_{k}\right)\right) 1_{\left\{X_{k}=x_{k}^{i}\right\}} \\
& \text { and } \\
q_{k}= & \bar{Q}^{k}-Q_{k}=\sum_{i=1}^{N_{k}} \pi_{F^{1}, i_{0}, i}^{0, k-1} \frac{1}{p_{k}^{i}} \mu_{k} 1_{\left\{X_{k}=x_{k}^{i}\right\}}
\end{aligned}
$$

Since $\pi_{F^{1}, i_{0}, i}^{0, k-1} \leq \Pi_{j=1}^{k}\left[F_{j}\right]_{1} p_{k}^{i}$ it follows that $\left|q_{k}\right| \leq \Pi_{j=1}^{k}\left[F_{j}\right]_{1}\left|\mu_{k}\right|$ and so

$$
\left\|Z-\sum_{k=0}^{n-1} \bar{Q}^{k}\right\|_{2} \leq \sum_{k=0}^{n}\left\|q_{k}\right\|_{2} \leq \sum_{k=0}^{n} \Pi_{i=1}^{k+1}\left[F_{i}\right]_{1}\left\|\mu_{k}\right\|_{2} .
$$

So the quantity to be computed is now $\left\|\sum_{k=0}^{n-1} \bar{Q}^{k}\right\|_{2}$. Since $E\left(\bar{Q}^{k} \mid \mathcal{F}_{k}\right)=0$, we have $E\left(\bar{Q}^{k} \bar{Q}^{p}\right)=0$ for $k \neq p$ and so $\left\|\sum_{k=0}^{n-1} \bar{Q}^{k}\right\|_{2}^{2}=\sum_{k=0}^{n-1} E\left|\bar{Q}^{k}\right|^{2}$. On the other hand

$$
\begin{aligned}
E\left|\bar{Q}^{k}\right|^{2} & =\sum_{i=1}^{N_{k}} E \mid \pi_{F^{1}, i_{0}, i}^{0, k-1} \frac{1}{p_{k}^{i}}\left(\widehat{F}_{k+1}\left(X_{k+1}\right)-\left.E\left(\widehat{F}_{k+1}\left(X_{k+1}\right) \mid \mathcal{F}_{k}\right)\right|^{2} 1_{\left\{X_{k}=x_{k}^{i}\right\}}\right. \\
& =\sum_{i=1}^{N_{k}}\left(\pi_{F^{1}, i_{0}, i}^{0, k-1} \frac{1}{p_{k}^{i}}\right)^{2} E\left(\widehat{F}_{k+1}^{2}\left(X_{k+1}\right)-\left(E\left(\widehat{F}_{k+1}\left(X_{k+1}\right) \mid \mathcal{F}_{k}\right)\right)^{2}\right) 1_{\left\{X_{k}=x_{k}^{i}\right\}} \\
& \leq\left(\Pi_{j=1}^{k}\left[F_{j}\right]_{1}\right)^{2} E\left(\widehat{F}_{k+1}^{2}\left(X_{k+1}\right)-\left(E\left(\widehat{F}_{k+1}\left(X_{k+1}\right) \mid \mathcal{F}_{k}\right)\right)^{2}\right) .
\end{aligned}
$$

By the very definition of $\widehat{u}_{k}, E\left(\widehat{F}_{k+1}\left(X_{k+1}\right) \mid X_{k}\right)=\widehat{u}_{k}\left(X_{k}\right)$, so that

$$
\begin{aligned}
E\left|\bar{Q}^{k}\right|^{2} \leq & \Pi_{j=1}^{k}\left[F_{j}\right]_{1}^{2} E\left(\widehat{F}_{k+1}^{2}\left(X_{k+1}\right)-\widehat{u}_{k}\left(X_{k}\right)^{2}\right) \\
& \left.\left.+\Pi_{j=1}^{k}\left[F_{j}\right]_{1}^{2} E\left(E\left(\widehat{F}_{k+1}\left(X_{k+1}\right) \mid \mathcal{X}_{k}\right)\right)^{2}-E\left(\widehat{F}_{k+1}\left(X_{k+1}\right) \mid \mathcal{F}_{k}\right)\right)^{2}\right) \\
\leq & \Pi_{j=1}^{k}\left[F_{j}\right]_{1}^{2} E\left(\widehat{F}_{k+1}^{2}\left(X_{k+1}\right)-\widehat{u}_{k}\left(X_{k}\right)^{2}\right)+\Pi_{i=1}^{k+1}\left[F_{i}\right]_{1}^{2}\left\|F_{k+1}\right\|_{\infty}\left\|\mu_{k}\right\|_{2} .
\end{aligned}
$$

Summing over $k$ we obtain the upper bound is completed. The proof of the lower bound is the same: one just employ the minoration $\pi_{F^{1}, i_{0}, i}^{0, k} \geq \Pi_{i=1}^{k+1}\left\{F_{i}\right\}_{1} p_{k+1}^{i}$ instead of the majoration $\pi_{F^{1}, i_{0}, i}^{0, k} \leq \Pi_{i=1}^{k+1}\left[F_{i}\right]_{1} p_{k+1}^{i}$.

We give now a setting in which the "Markov error" considered above may be computed in a reasonable way. We assume that an $E$ - valued Markov chain $\left(\bar{X}_{k}\right)_{k \geq 0}$ with respect to some filtration $\left(\mathcal{F}_{k}\right)_{k \geq 0}$ is given and we construct $X_{k}=\theta_{k}\left(\bar{X}_{k}\right)$ where $\theta_{k}: E \rightarrow E, k=1, \ldots, n$ are some measurable functions. The example which we have in mind is the quantization

INRIA 
procedure which amounts to construct $X_{k}$ as the projection of $\bar{X}_{k}$ on a given grid. We also define

$$
\left.\Phi_{k}(x)=E\left(\widehat{F}_{k+1}\left(\bar{X}_{k+1}\right) \mid \bar{X}_{k}=x\right)\right) .
$$

Moreover we assume that $E$ is a normed space and $\Phi_{k}$ is Lipschitz continuous. We denote the Lipschitz constant by $\left[\Phi_{k}\right]_{1}$ and $[\Phi]_{1}=: \max _{k=1, n}\left[\Phi_{k}\right]_{1}$. We have to control the Markov error for the chain $\left(X_{k}\right)_{k}$. We write

$$
\begin{aligned}
\mu_{k}= & E\left(\widehat{F}_{k+1}\left(X_{k+1}\right)-\widehat{F}_{k+1}\left(\bar{X}_{k+1}\right) \mid X_{k}\right)-E\left(\widehat{F}_{k+1}\left(X_{k+1}\right)-\widehat{F}_{k+1}\left(\bar{X}_{k+1}\right) \mid \mathcal{F}_{k}\right) \\
& +\left(E\left(\widehat{F}_{k+1}\left(\bar{X}_{k+1}\right) \mid X_{k}\right)-E\left(\widehat{F}_{k+1}\left(\bar{X}_{k+1}\right) \mid \mathcal{F}_{k}\right)\right) .
\end{aligned}
$$

Moreover using the Markov property of $\left(\bar{X}_{k}\right)_{k \geq 0}$ first and the fact that $X_{k}$ is $\sigma\left(\bar{X}_{k}\right)$ measurable then,

$$
\begin{aligned}
E\left(\widehat{F}_{k+1}\left(\bar{X}_{k+1}\right) \mid\right. & \left.X_{k}\right)-E\left(\widehat{F}_{k+1}\left(\bar{X}_{k+1}\right) \mid \mathcal{F}_{k}\right) \\
= & E\left(E\left(\widehat{F}_{k+1}\left(\bar{X}_{k+1}\right) \mid \bar{X}_{k}\right) \mid X_{k}\right)-E\left(\widehat{F}_{k+1}\left(\bar{X}_{k+1}\right) \mid \bar{X}_{k}\right) \\
= & E\left(\Phi_{k}\left(\bar{X}_{k}\right) \mid X_{k}\right)-\Phi_{k}\left(\bar{X}_{k}\right) \\
= & E\left(\Phi_{k}\left(\bar{X}_{k}\right)-\Phi_{k}\left(X_{k}\right) \mid X_{k}\right)+\Phi_{k}\left(X_{k}\right)-\Phi_{k}\left(\bar{X}_{k}\right) .
\end{aligned}
$$

It follows that

$$
\left\|\mu_{k}\right\|_{2} \leq 2\left[\widehat{F}_{k+1}\right]_{1}\left\|X_{k+1}-\bar{X}_{k+1}\right\|_{2}+2\left[\Phi_{k}\right]_{1}\left\|X_{k}-\bar{X}_{k}\right\|_{2} .
$$

This together with the above proposition give

Proposition 4 Suppose that $\left.\bar{X}_{k}\right)_{k \in N}$ is a Markov chain such that $\Phi_{k}$ is Lipschitz continuous and $X_{k}=\theta_{k}\left(\bar{X}_{k}\right)$ for some measurable functions $\theta_{k}$.

i) Suppose that (4) holds. Then

$$
\begin{aligned}
\sigma_{n, N} \leq & \left(\sum_{k=0}^{n-1} \Pi_{r=1}^{k+1}\left[F_{r}\right]_{1}^{2} E\left(\widehat{F}_{k+1}^{2}\left(X_{k+1}\right)-\widehat{u}_{k}^{2}\left(X_{k}\right)\right)\right)^{1 / 2} \\
& +4\left([\widehat{F}]_{1}+[\Phi]_{1}\right) \sum_{k=0}^{n} \Pi_{i=1}^{k+1}\left[F_{i}\right]_{1}\left\|F_{k+1}\right\|_{\infty}\left\|X_{k}-\bar{X}_{k}\right\|_{2}
\end{aligned}
$$

ii) Suppose that (21) holds also. Then

$$
\begin{aligned}
\sigma_{n, N} \geq & \left(\sum_{k=0}^{n-1} \Pi_{r=1}^{k+1}\left\{F_{r}\right\}_{1}^{2} E\left(\widehat{F}_{k+1}^{2}\left(X_{k+1}\right)-\widehat{u}_{k}^{2}\left(X_{k}\right)\right)\right)^{1 / 2} \\
& -2\left([\widehat{F}]_{1}+[\Phi]_{1}\right) \sum_{k=0}^{n} \Pi_{i=1}^{k+1}\left[F_{i}\right]_{1}\left\|F_{k+1}\right\|_{\infty}\left\|X_{k}-\bar{X}_{k}\right\|_{2} .
\end{aligned}
$$




\section{The CLT for semi linear PDE's}

Our main interest in this paper concerns semi linear PDE's i.e (29) bellow and semi linear PDE's with reflection, i.e (30) bellow. We summarize in this section the results obtained in [B.P.1], [B.P.2] for the approximation scheme $\widehat{u}_{k}$ of the solution $u$ of this equation.

In order to construct our algorithm we consider the diffusion process $\left(X_{t}\right)_{t \geq 0}$ of infinitesimal operator $L$ (which appear in the $P D E$ ) and we denote by $\bar{X}_{k}$ the Euler Scheme of step $h=\frac{T}{n}$ (see [B.P.1] for details). The diffusion process takes values in $R^{d}$ so the space under consideration is now $E=R^{d}$. Moreover for each $k=1, \ldots, n$ we construct a grid $\Gamma_{k}=\left\{x_{k}^{1}, \ldots x_{k}^{N_{k}}\right\}$ and we denote $I_{k}^{i}=\left\{x:\left|x_{k}^{i}-x\right|=\min _{j=1, N_{k}}\left|x_{k}^{j}-x\right|\right\}$ the so called Voronoi tassel of $x_{k}^{i}$. Then $X_{k}$ is the projection of $\bar{X}_{k}$ on the grid $\Gamma_{k}, i . e X_{k}=x_{k}^{i}$ if $\bar{X}_{k}^{i} \in I_{k}^{i}$. Since $\left(\bar{X}_{k}\right)_{k=1, n}$ is a Markov chain we are in the frame presented in the previous section. We recall also that the grid $\Gamma_{k}$ is chosen to be optimal in the sense that the law of the projection $X_{k}$ on $\Gamma_{k}$ is "closer" to the law of $\bar{X}_{k}$ then the projection on any other grid $\Gamma_{k}^{\prime}$. This is discussed in detail in [B.P.1] but is not the subject now. Here we just assume that a fixed grid $\Gamma_{k}$ is given and we compute our algorithm based on this grid.

The quality of our evaluations depends on the regularity of the problem in two senses. First of all, when dealing with a general diffusion process, the first step to be done in order to construct an approximation scheme is to replace the underlying diffusion by the Euler Scheme. This is necessary in order to be able to simulate the pathes. But in some special cases - for example if the diffusion process is the Brownian motion itself or the geometrical Brownian motion, as in the Black and Sholes model - one can simulate the diffusion directly and there is no need to replace the diffusion by the Euler scheme (in fact they coincide). The analysis of the error turns out to be more precise in this last case. Secondly, the coefficient $f$ and the obstacle $h$ may be just Lipschitz continuous or may have some more regularity properties, and of course we get better results in the second case. So we shall distinguish two cases.

Case 1. $\bar{X}_{k}$ is the Euler Scheme and $f$ and $h$ are Lipschitz continuous.

Case 2. $\bar{X}_{k}$ is the diffusion process itself at time $t_{k}=k T / n, y \rightarrow f(t, x, y)$ si of class $C_{b}^{2}$, and $x \rightarrow h(t, x)$ is semi - convex, uniformly with respect to the time variable i.e

$$
h(t, y)-h(t, x) \geq(\delta(t, x), y-x)+\rho(t, x)|y-x|^{2}
$$

where $\delta$ and $\rho$ are some bounded functions.

Let us now present the error evaluations obtained in [B.P.1]. We recall that in [B.P.1] we considered that the total number of points in the time - space grid $\bar{N}=: \sum_{k=1}^{n} N_{k}$ is given (the complexity of our algorithm is proportional to $\bar{N}$ so this is the natural parameter to be fixed from the beginning) and we look for the optimal choice of the number of time

steps $n$ and of the number of points $N_{k}$ in the layer $k$ to be used. A sorrow analysis of this optimization problem is given in [B.P.1] Section II. 2.2. This analysis is based on the 
structure of the error on one hand and on the evaluation of the error in the quantization procedure (the Theorem of Buckley and Wise see [B.P.1] or $[G . L]$ ) on the other hand. In our frame some more errors come on and this optimization becomes rather difficult to active.. So we give up and we decide to take the same number $N$ of points on each layer. Recall that $\bar{N}$ is given. Then, according to the computations in [B.P.1], we have to choose $n=\bar{N}^{2 /(3 d+2)}$ in the Case 1 and $n=\bar{N}^{1 /(2 d+1)}$ in the Case 2. In both cases we take $N_{k}=N=\bar{N} / n$. This amounts to take $N_{k}=N=n^{3 d / 2}$ in Case 1 and $N_{k}=n^{2 d}$ in Case 2. Now on we assume that this choice is done and we express the error bounds in terms of $\frac{1}{n}$. In Case 1 we obtain (see $[B . P .1]$ and $[B . P .2])$

$$
\begin{aligned}
E \max _{k=0, n}\left|u\left(t_{k}, X_{k}\right)-\widehat{u}_{k}\left(X_{k}\right)\right| & \leq \frac{C}{\sqrt{n}} \\
\left\|X_{k}-\bar{X}_{k}\right\|_{4} & \leq \frac{C}{N^{1 / d}} \leq \frac{C}{n \sqrt{n}} .
\end{aligned}
$$

and in the Case 2

$$
\begin{aligned}
E \max _{k=0, n}\left|u\left(t_{k}, X_{k}\right)-\widehat{u}_{k}\left(X_{k}\right)\right| & \leq \frac{C}{n}, \\
\left\|X_{k}-\bar{X}_{k}\right\|_{4} & \leq \frac{C}{N^{1 / d}} \leq \frac{C}{n^{2}} .
\end{aligned}
$$

These evaluations hold true for both semi linear PDE's with and without reflection. Although our main interest concerns obstacle problems we begin with $P D E^{\prime} s$ without obstacle because this case is simpler and we are able to aciev a more precise analysis.

\subsection{Semi linear PDE's without reflection.}

In this section we discuss the algorithm corresponding to the semi linear $P D E$

$$
\left(\partial_{t}+L\right) u+f(t, x, u)=0, \quad u_{T}=g .
$$

The function $F_{k}$ is now $F_{k}(x, y)=y+\frac{T}{n} f\left(t_{k}, x, y\right)$. We assume that $g$ is bounded and that $y \rightarrow f(t, x, y)$ is twice differentiable and the first and the second derivatives are bounded, uniformly with respect to $t$ and $x$. Since the problem is simpler, one can prove (28) even in the case when $X_{k}$ is the Euler Scheme. It is also easy to check that $\left\|\widehat{u}_{k}\right\|_{\infty} \leq C\|g\|_{\infty}$ and so $\left\|\widehat{F}_{k}\right\|_{\infty} \leq C\left(1+\|g\|_{\infty}\right)$. Moreover $F_{k}^{1}(x, y)=\frac{\partial F_{k}}{\partial y}(x, y)=1+\frac{T}{n} \frac{\partial f}{\partial y}\left(t_{k}, x, y\right)$ so that $[F]_{1} \leq 1+\frac{T}{n}[f]_{1}$ and $[F]_{1}^{n} \leq \exp \left(T[f]_{1}\right)$. 
Let us now evaluate the variance $\sigma_{n, N}$. We have $[F]_{1} \leq 1+\frac{T}{n}[f]_{1}$ and $\{F\}_{1} \geq 1-\frac{T}{n}[f]_{1}$ so, using (24), (25) and (28) we obtain

$$
\begin{aligned}
& \sum_{k=0}^{n-1}\left(1-\frac{T}{n}[f]_{1}\right)^{k}\left(E \widehat{F}_{k+1}^{2}\left(X_{k+1}\right)-E \widehat{u}_{k}^{2}\left(X_{k}\right)\right)-\frac{C}{n} \\
\leq & \sigma_{n, N} \leq \sum_{k=0}^{n-1}\left(1+\frac{T}{n}[f]_{1}\right)^{k}\left(E \widehat{F}_{k+1}^{2}\left(X_{k+1}\right)-E \widehat{u}_{k}^{2}\left(X_{k}\right)\right)+\frac{C}{n} .
\end{aligned}
$$

We have $\widehat{F}_{k+1}^{2}=\widehat{u}_{k+1}^{2}+\frac{T}{n} \widehat{u}_{k+1} f\left(t_{k+1}, ., \widehat{u}_{k+1}\right)+\frac{T^{2}}{n^{2}} f^{2}\left(t_{k+1}, . . \widehat{u}_{k+1}\right)$.

We compute first

$$
\begin{aligned}
& \sum_{k=0}^{n-1}\left(1+\frac{T}{n}[f]_{1}\right)^{k}\left(E \widehat{u}_{k+1}^{2}\left(X_{k+1}\right)-E \widehat{u}_{k}^{2}\left(X_{k}\right)\right) \\
= & \left(1+\frac{T}{n}[f]_{1}\right)^{n-1} E g^{2}\left(X_{n}\right)-\widehat{u}_{0}^{2}\left(x_{0}\right)-\sum_{k=1}^{n-2}\left(1+\frac{T}{n}[f]_{1}\right)^{k-1} \frac{T}{n}[f]_{1} E \widehat{u}_{k}^{2}\left(X_{k}\right) .
\end{aligned}
$$

Using (28) the above term is equal to

$$
\lambda_{n}+\left(1+\frac{T}{n}[f]_{1}\right)^{n-1} E g^{2}\left(X_{T}\right)-u_{0}^{2}\left(x_{0}\right)-\sum_{k=1}^{n-2}\left(1+\frac{T}{n}[f]_{1}\right)^{k-1} \frac{T}{n}[f]_{1} E u^{2}\left(t_{k}, X_{t_{k}}\right)
$$

where $\left|\lambda_{n}\right| \leq C / n$. Moreover, standard computations show that the above term is still equal to

$$
\lambda_{n}^{\prime}+e^{T[f]_{1}} E g^{2}\left(X_{T}\right)-u_{0}^{2}\left(x_{0}\right)-[f]_{1} \int_{0}^{T} e^{t[f]_{1}} E u^{2}\left(t, X_{t}\right) d t
$$

with $\left|\lambda_{n}^{\prime}\right| \leq C / n$. The same reasoning shows that the sum corresponding to $\frac{T}{n} \widehat{u}_{k+1} f\left(t_{k+1}, . . \widehat{u}_{k+1}\right)$ is equal to

$$
\lambda_{n}^{\prime \prime}+2 \int_{0}^{T} e^{t[f]_{1}} E\left(u_{t}\left(X_{t}\right) f\left(t, X_{t}, u\left(t, X_{t}\right)\right) d t\right.
$$

with $\left|\lambda_{n}^{\prime \prime}\right| \leq C / n$. Finally the term corresponding to $\frac{T^{2}}{n^{2}} f^{2}$ is dominated by $\frac{1}{n}\|f\|_{\infty}^{2}$ so we have proved that $\sigma_{n, N} \leq \bar{\sigma}+\frac{C}{n}$ with

$$
\bar{\sigma}=e^{T[f]_{1}} P_{T} g^{2}\left(x_{0}\right)-u_{0}^{2}\left(x_{0}\right)+\int_{0}^{T} e^{t[f]_{1}} P_{t}\left(2 u_{t} f\left(t, ., u_{t}\right)-[f]_{1} u_{t}^{2}\right)\left(x_{0}\right) d t
$$

where $P_{t}$ is the semigroup of the diffusion process. In the same way one proves that $\sigma_{n, N} \geq$ $\underline{\sigma}-\frac{C}{n}$ with

$$
\underline{\sigma}=e^{-T[f]]_{1}} P_{T} g^{2}\left(x_{0}\right)-u_{0}^{2}\left(x_{0}\right)+\int_{0}^{T} e^{-t[f]_{1}} P_{t}\left(2 u_{t} f\left(t, ., u_{t}\right)+[f]_{1} u_{t}^{2}\right)\left(x_{0}\right) d t .
$$

So we have proved

INRIA 
Theorem 1 Let $u$ be the solution of the semi-linear PDE (29), let $\widehat{u}$ be computed by the algorithm (2) and $\bar{u}$ be computed by the algorithm (3). Suppose that the final condition $g$ is bounded and the coefficient $f$ is bounded, twice differentiable in the variable $y$, with bounded derivatives of first and second order. Then $\left|u_{0}\left(x_{0}\right)-\widehat{u}_{0}\left(x_{0}\right)\right| \leq C / n$ and

$$
\sqrt{M}\left(\bar{u}_{0}\left(x_{0}\right)-\widehat{u}_{0}\left(x_{0}\right)\right) \rightarrow \Theta \sim N\left(0, \sigma_{n, N}\right) \quad \text { with } \quad \underline{\sigma}-\frac{C}{n} \leq \sigma_{n, N} \leq \bar{\sigma}+\frac{C}{n} .
$$

\subsection{The CLT for semi-linear PDE's with reflection.}

We discuss now the algorithm corresponding to the semi linear $P D E$ with obstacle

$$
\max \left\{h_{t},\left(\partial_{t}+L\right) u+f(t, x, u)\right\}=0, \quad u_{T}=h_{T}
$$

that is

$$
\begin{aligned}
& \widehat{u}_{n}\left(x_{n}^{i}\right)=h\left(t_{n}, x_{n}^{i}\right) \\
& \widehat{u}_{k}\left(x_{k}^{i}\right)=\bar{E}_{i}^{k}\left(\max \left\{h\left(t_{k+1}, .\right), \widehat{u}_{k+1}(.)+\frac{T}{n} f\left(t_{k}, ., \widehat{u}_{k+1}(.)\right)\right)\right.
\end{aligned}
$$

The function $F_{k}$ is now $F_{k}(x, y)=\max \left\{h\left(t_{k}, x\right), y+\frac{T}{n} f\left(t_{k}, x, y\right)\right\}$.

Remark 1 The algorithm here is slightly different from the one usually considered in the Dynamical Programing Principle for the Snell envelope which is $v_{n}\left(x_{n}^{i}\right)=h_{n}\left(x_{n}^{i}\right), v_{k}\left(x_{k}^{i}\right)=$ $\max \left\{h_{k}\left(x_{k}^{i}\right), \bar{E}_{i}^{k}\left(v_{k+1}()+.\frac{T}{n} f\left(t_{k}, ., v_{k+1}\right)\right)\right.$ with $h_{k}(x)=h\left(t_{k}, x\right)$. So we changed the order of the expectation and of the maximum. This is convenient in order to fit in the frame discussed in Section 2. Let us see that this is not a significant change. This is quite clear if $f$ is null (we treat just an optimal stopping problem). In fact, if one denotes $w_{k}=$ $\max \left(h_{k}, \widehat{u}_{k}\right)$ then $w_{n}=h_{n}$ and $w_{k}=\max \left(h_{k}, \bar{E}^{k}\left(\max \left\{h_{k+1}, \widehat{u}_{k+1}\right\}\right)\right)=\max \left(h_{k}, \bar{E}^{k} w_{k+1}\right)$. So $w$ is constructed in the same way as $v$ and consequently $w_{k}=v_{k}$. We conclude that $v_{0}=w_{0}=\max \left(h_{0}, \widehat{u}_{0}\right)$. If $f$ is not null a supplementary error of order $\frac{1}{n}$ comes on.

We assume that $g=: h_{T}$ is bounded and that $y \rightarrow f(t, x, y)$ is Lipschitz continuous.. Let us now evaluate the variance $\sigma_{n, N}$. We denote

$$
\begin{aligned}
b_{k}(x) & =\widehat{u}_{k}(x)+\frac{T}{n} f\left(t_{k}, x, \widehat{u}_{k}(x)\right), \\
c_{k}(x) & =\left(h_{k}(x)-b_{k}(x)\right) 1_{\left\{h_{k}>b_{k}\right\}}
\end{aligned}
$$

such that

$$
\widehat{F}_{k}(x)=\max \left\{h_{k}(x), b_{k}(x)\right\}=b_{k}(x)+c_{k}(x) .
$$

Lemma 1 i) Suppose that $h$ is Lipschitz continuous. Then

$$
E_{i_{0}}^{0, k} c_{k}^{2} \leq \frac{C}{n} .
$$

$\mathrm{RR} \mathrm{n}^{\circ} 4629$ 
ii) Suppose that h is Lipschitz continuous and semi - convex. Then

$$
E_{i_{0}}^{0, k} c_{k}^{2} \leq \frac{C}{n^{2}}
$$

Proof. We prove just ii) (the first point is analogous but simpler) so we suppose that $h$ is semi - convex. Using the definition of $\widehat{u}_{k}$,

$$
\begin{aligned}
b_{k}\left(x_{k}^{i}\right)= & E_{i}^{k}\left(\max \left\{h_{k+1}, \widehat{u}_{k+1}+\frac{T}{n} f\left(t_{k+1}, ., \widehat{u}_{k+1}(.)\right)\right\}\right) \\
& +\frac{T}{n} f\left(t_{k}, x_{k}^{i}, \widehat{u}_{k}\left(x_{k}^{i}\right)\right) \\
\geq & E_{i}^{k} h_{k+1}+\frac{T}{n} f\left(t_{k}, x_{k}^{i}, \widehat{u}_{k}\left(x_{k}^{i}\right)\right)
\end{aligned}
$$

so that

$$
\begin{aligned}
0 & \leq c_{k}\left(x_{k}^{i}\right) \leq\left(h_{k}\left(x_{k}^{i}\right)-E_{i}^{k} h_{k+1}-\frac{T}{n} f\left(t_{k}, x_{k}^{i}, \widehat{u}_{k}\left(x_{k}^{i}\right)\right)\right) 1_{\left\{h_{k}>b_{k}\right\}}\left(x_{k}^{i}\right) \\
& \leq E\left(h_{k}\left(x_{k}^{i}\right)-h_{k+1}\left(X_{k+1}\right) \mid X_{k}=x_{k}^{i}\right) 1_{\left\{h_{k}>b_{k}\right\}}\left(x_{k}^{i}\right)+\frac{T}{n}\|f\|_{\infty} \\
& \leq E\left(h_{k}\left(x_{k}^{i}\right)-h_{k}\left(X_{k+1}\right) \mid X_{k}=x_{k}^{i}\right) 1_{\left\{h_{k}>b_{k}\right\}}\left(x_{k}^{i}\right)+\frac{T}{n}\left(\|f\|_{\infty}+[h]_{1}\right) \\
& \leq E\left(\left(\delta\left(t_{k}, x_{k}^{i}\right),\left(X_{k+1}-x_{k}^{i}\right)\right) \mid X_{k}=x_{k}^{i}\right) 1_{\left\{h_{k}>b_{k}\right\}}\left(x_{k}^{i}\right) \\
+\|\rho\|_{\infty} E\left(\left|X_{k+1}-x_{k}^{i}\right|^{2}\right. & \left.\mid X_{k}=x_{k}^{i}\right)+\frac{T}{n}\left(\|f\|_{\infty}+[h]_{1}\right) .
\end{aligned}
$$

Using (28)

$$
E\left|E\left(\left|X_{k+1}-x_{k}^{i}\right|^{2} \mid X_{k}=x_{k}^{i}\right)\right|^{2} \leq E\left|X_{k+1}-X_{k}\right|^{4} \leq E\left|\bar{X}_{k+1}-\bar{X}_{k}\right|^{4}+\frac{C}{n^{8}} \leq \frac{C}{n^{2}} .
$$

It follows that

$$
\begin{aligned}
E_{i_{0}}^{0, k} c_{k}^{2} & =E c_{k}^{2}\left(X_{k}\right) \leq 2 E\left(\left|E\left(\left(\delta\left(t_{k}, X_{k}\right),\left(X_{k+1}-X_{k}\right)\right) \mid X_{k}\right)\right|^{2}+\frac{C}{n^{2}}\right. \\
& \leq 2 E\left(E\left(\left(\delta\left(t_{k}, X_{k}\right),\left(\bar{X}_{k+1}-\bar{X}_{k}\right)\right) \mid X_{k}\right)\right)^{2}+\frac{C}{n^{2}} \\
& =2 E\left(E\left(E\left(\left(\delta\left(t_{k}, X_{k}\right),\left(\bar{X}_{k+1}-\bar{X}_{k}\right)\right) \mid F_{k}\right) \mid X_{k}\right)\right)^{2}+\frac{C}{n^{2}} .
\end{aligned}
$$

Since the martingale part of $\bar{X}_{k+1}-\bar{X}_{k}$ cancels when we take conditional expectation with respect to $F_{k}$, this term is dominated by $C / n^{2}$ where $C$ is a constant which depends on the coefficients of the diffusion process. So the proof is completed.

We are now able to give the evaluation of $\sigma_{n, N}$.

INRIA 
Proposition 5 i) I $n$ the Case 1 we have

$$
\sigma_{n, N}^{2} \leq C \sqrt{n}
$$

ii) In the Case 2

$$
\sigma_{n, N}^{2} \leq \frac{C}{n}+\widetilde{\sigma}^{2}
$$

with

$$
\widetilde{\sigma}^{2}=e^{T[f]_{1}} P_{T} g^{2}\left(x_{0}\right)-u_{0}\left(x_{0}\right)+C \int_{0}^{T} e^{t[f]_{1}} P_{t}\left(u_{t}^{2}+u_{t} f\left(t, ., u_{t}\right)\right)\left(x_{0}\right) d t
$$

where $C$ is a constant depending on the diffusion process.

Proof. We write

$$
\begin{aligned}
E \widehat{F}_{k}^{2}\left(X_{k}\right) & =E\left(b_{k}+c_{k}\right)^{2}\left(X_{k}\right) \\
& \leq E b_{k}^{2}\left(X_{k}\right)+2\left(E b_{k}^{2}\left(X_{k}\right)\right)^{1 / 2}\left(E c_{k}^{2}\left(X_{k}\right)\right)^{1 / 2}+E c_{k}^{2}\left(X_{k}\right) .
\end{aligned}
$$

In the Case 1 (31) permits to dominate the above term by $E b_{k}^{2}+C / \sqrt{n}$, and since $n \times C / \sqrt{n}=C \sqrt{n}$ we get $\sqrt{n}$ in the $R H S$ of (33).

Assume now that we are in the Case 2, we may use (32) in order to get $E c_{k}^{2} \leq C / n^{2}$ and consequently

$$
E \widehat{F}_{k}^{2}\left(X_{k}\right) \leq E b_{k}^{2}\left(X_{k}\right)+\frac{C}{n}\left(E b_{k}^{2}\left(X_{k}\right)\right)^{1 / 2}+\frac{C}{n^{2}} .
$$

We employ (28) in order to obtain

$$
\left(E b_{k}^{2}\left(X_{k}\right)\right)^{1 / 2} \leq \frac{C}{n}+\left(E\left|\widehat{u}_{k}\left(X_{k}\right)\right|^{2}\right)^{1 / 2} \leq \frac{C}{n}+\left(E\left|u\left(t_{k}, x_{k}\right)\right|^{2}\right)^{1 / 2}
$$

so that

$$
E \widehat{F}_{k}^{2}\left(X_{k}\right) \leq E b_{k}^{2}\left(X_{k}\right)+\frac{C}{n}\left(P_{t_{k}} u_{t_{k}}^{2}\left(x_{0}\right)\right)^{1 / 2}+\frac{C}{n^{2}} .
$$

We employ now (24) in order to obtain

$$
\begin{aligned}
\sigma_{n, N}^{2} & \leq \frac{C}{n}+\sum_{k=1}^{n-1} e^{t_{k}[f]_{1}}\left(E\left(\widehat{F}_{k+1}\right)^{2}\left(X_{k+1}\right)-E\left(\widehat{u}_{k}^{2}\left(X_{k}\right)\right)\right) \\
& \leq \frac{C}{n}+\frac{C}{n} \sum_{k=1}^{n-1} e^{t_{k}[f]_{1}}\left(P_{t_{k}} u_{t_{k}}^{2}\left(x_{0}\right)\right)^{1 / 2}+\sum_{k=1}^{n-1} e^{t_{k}[f]_{1}}\left(E b_{k+1}^{2}\left(X_{k}\right)-E\left(\widehat{u}_{k}^{2}\left(X_{k}\right)\right)\right.
\end{aligned}
$$

$\mathrm{RR} \quad \mathrm{n}^{\circ} 4629$ 
Since $b_{k+1}^{2}=\widehat{u}_{k+1}^{2}+\frac{2 T}{n} \widehat{u}_{k+1} f\left(t_{k+1}, . . \widehat{u}_{k+1}\right)+\frac{4 T^{2}}{n^{2}} f^{2}\left(t_{k+1}, ., \widehat{u}_{k+1}\right)$ we have

$$
\begin{aligned}
& \sum_{k=1}^{n-1} e^{t_{k}[f]_{1}}\left(E b_{k+1}^{2}\left(X_{k}\right)-E\left(\widehat{u}_{k}^{2}\left(X_{k}\right)\right)\right. \\
\leq & \frac{C}{n}+\sum_{k=1}^{n-1} e^{t_{k}[f]_{1}}\left(E\left(\widehat{u}_{k+1}\right)^{2}\left(X_{k+1}\right)-E\left(\widehat{u}_{k}^{2}\left(X_{k}\right)\right)\right) \\
& +\frac{2 T}{n} \sum_{k=1}^{n-1} e^{t_{k}[f]_{1}}\left(E \left(\widehat{u}_{k+1} f\left(t_{k+1}, ., \widehat{u}_{k+1}\right)\left(X_{k+1}\right)\right.\right. \\
= & \frac{C}{n}+E h_{T}^{2}\left(X_{T}\right)-\widehat{u}_{0}^{2}\left(x_{0}\right) \\
& +\frac{2 T}{n} \sum_{k=1}^{n-1} e^{t_{k}[f]_{1}} E\left(\widehat{u}_{k+1} f\left(t_{k+1}, ., \widehat{u}_{k+1}\right)\left(X_{k+1}\right)\right. \\
\leq & \frac{C}{n}+P_{T} h_{T}^{2}\left(x_{0}\right)-u_{0}^{2}\left(x_{0}\right)+\frac{2 T}{n} \sum_{k=1}^{n-1} e^{t_{k}[f]_{1}} P_{t_{k+1}}\left(u_{t_{k+1}} f\left(t_{k+1}, ., u_{t_{k+1}}\right)\left(x_{0}\right) .\right.
\end{aligned}
$$

Finally, passing from the Riemann sums to Lebesgue's integrals with respect to the time, the proof is completed.

Let us now conclude.

Theorem 2 There exists a bounded random variable $m_{n}^{M}$ such that

$$
\begin{aligned}
\text { i) }\left|m_{n}^{M}(\omega)\right| & \leq \frac{C}{n}, \forall \omega \\
\text { ii) } \lim _{n} \sqrt{M}\left(\widehat{u}_{0}\left(x_{0}\right)-\bar{u}_{0}\left(x_{0}\right)-m_{n}^{M}\right) & =\Theta \sim N\left(0, \sigma_{n}\right)
\end{aligned}
$$

in low and $\sigma_{n}^{2} \leq C \sqrt{n}$ in Case 1 and

$$
\sigma_{n}^{2} \leq \frac{C}{n}+e^{T[f]_{1}} P_{T} g^{2}\left(x_{0}\right)-u_{0}\left(x_{0}\right)+C \int_{0}^{T} e^{t[f]_{1}} P_{t}\left(u_{t}^{2}+u_{t} f\left(t, ., u_{t}\right)\right)\left(x_{0}\right) d t
$$

in Case 2.

Proof. We employ Proposition 2 with $\varepsilon=\varepsilon_{n}=\frac{1}{n^{2}}$. The absolute value of $m_{n}^{M}=$ : $m_{n, N}^{\varepsilon_{n}}$ is dominated by $2 n\left(1+\frac{T}{n}[f]_{1}\right)^{n} \varepsilon_{n} \leq 2 n e^{T[f]_{1}} / n^{2}=C / n$. Moreover we obtain the convergence in ii) with $\sigma_{n}=\sigma_{n, N}^{\varepsilon_{n}}$. Let us check that $\sigma_{n}$ satisfies (35). Since $\left[F_{k}^{\varepsilon_{n}}\right]_{1} \leq\left[F_{k}\right]_{1}$ and $\left\|F_{k}^{\varepsilon_{n}}\right\|_{\infty} \leq\left\|F_{k}\right\|_{\infty}$ we may employ (24) in order to obtain

$$
\sigma_{n}^{2} \leq \frac{C}{n}+\sum_{k=0}^{n-1} \Pi_{r=1}^{k+1}\left[F_{r}\right]_{1}^{2} E\left(\widehat{F}_{k+1}^{2}\left(X_{k+1}\right)-\widehat{u}_{k}^{2}\left(X_{k}\right)\right) .
$$

The term in the $R H S$ of the above inequality is dominated (see the proof of Proposition 7 ) by the term in the $R H S$ of (35) and of the proof is completed.

INRIA 


\section{References}

[B.P.1] V. Bally, G. Pagès, A quantization algorithm for solving multi-dimensional Optimal Stopping problems, Preprint no 628, Laboratoire de Probabilitée et Modeles Aléatoires, Paris VI, 2000. To appear in Bernoulli.

[B.P.2] V. Bally, G. Pagès, Error analysis of the quantization algorithm for obstacle problems. Preprint no 642, Laboratoire de Probabilitée et Modeles Aléatoires, Paris VI, 2001. To appear in SPA.

[B.P.P] V. Bally, G. Pagès, J. Printemps, A stochastic quantization method for non linear problems Mont Carlo Methods and Applications, 7,no 1-2, pp 21-34, 2001.

[B.C.F.El-K] V Bally, M.E. Caballero, B. Fernandez, N. El-Karoui, Reflected BSDE's, PDE's and Variational Inequalities. Preprint 4455, INRIA, 2002

[B.L] A. Benssousan, J.L. Lions, Applications of Variational Inequalities in Stochastic Control, North Holland 1982.

[B.G] M. Broadie, P. Glasserman, Pricing American-style securities using simulation, J. of Economic Dynamics and Control, 21, 1323-1352, 1997.

[C.L.P] E. Clement, D. Lamberton, Ph. Protter An analysis of the Langstall - Schwartz algorithm for American option pricing, Preprint no 04/2000 Université Marne la Vallée, 2000. To appear in Finance and Stochastic, no 6, october 2002.

[El-K.P.Q] N. El-Karoui, S. Peng, M.C. Quenez, Backward Stochastic Differential Equations in finance, Math. Finance, 7, no 1,pp 1-71, 1997.

[El-K.K.P.P.Q] N. El-Karoui, C. Kapoudjan, E. Pardoux, S. Peng, M.C. Quenez, Reflected solutions of Backward Stochastic Differential Equationsand related obstacle problems for PDE's, The Ann. of Proba. , 25, no 2, pp 702-737, 1997.

[G.L] S. Graf, H. Luschgy, Fundation of quantization for random vectors, Lecture Notes in Mathematics no 730, Springer, 2000.

[L.S] F.A. Longstaff, E.S. Schwartz, Valuing American options by simulation: a simple less-squares approach, Review of Financial Studies, 14, 133-148, 2001.

[L.R] P.L. Lions, H. Régnier, Calcul des prix et des sénsibilités d'une option américaine par une methode de Monte Carlo, working paper.

[P.P] E. Pardoux, S. Peng, Adapted solutions for Backward Stochastic Differential Equations, Systems Control Letters,14, 55-61,1990. 


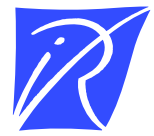

Unité de recherche INRIA Rocquencourt Domaine de Voluceau - Rocquencourt - BP 105 - 78153 Le Chesnay Cedex (France)

Unité de recherche INRIA Lorraine : LORIA, Technopôle de Nancy-Brabois - Campus scientifique 615, rue du Jardin Botanique - BP 101 - 54602 Villers-lès-Nancy Cedex (France)

Unité de recherche INRIA Rennes : IRISA, Campus universitaire de Beaulieu - 35042 Rennes Cedex (France)

Unité de recherche INRIA Rhône-Alpes : 655, avenue de l'Europe - 38330 Montbonnot-St-Martin (France)

Unité de recherche INRIA Sophia Antipolis : 2004, route des Lucioles - BP 93 - 06902 Sophia Antipolis Cedex (France)

INRIA - Domaine de Voluceau - Rocquencourt, BP 105 - 78153 Le Chesnay Cedex (France)

http://www.inria.fr

ISSN 0249-6399 